\title{
Conjunctival Melanoma Angiotropic Microsatellitosis: A Mechanism of Local Extravascular Migratory Metastasis
}

\author{
Jose J. Echegaray ${ }^{\mathrm{a}}$ Gabrielle Yeaney ${ }^{\mathrm{b}}$ Rachel Chen ${ }^{\mathrm{a}}$ Arun D. Singh ${ }^{\mathrm{a}}$ \\ ${ }^{a}$ Department of Ophthalmic Oncology, Cole Eye Institute, Cleveland Clinic, Cleveland, OH, USA; ${ }^{b}$ Department of \\ Pathology, Cleveland Clinic, Cleveland, OH, USA
}

\section{Established Facts}

- Local recurrence in conjunctival melanoma arises most commonly from remnant tumor present in margins after excision (contiguous recurrence) or is observed in cases with clinically unrecognized amelanotic melanoma beyond the tumor margins.

- Conjunctival melanoma is believed to achieve local metastasis via lymphatic dissemination and distant metastatic dissemination through hematogenous spread.

\section{Novel Insights}

- Local noncontiguous recurrence of conjunctival melanoma in absence of primary acquired melanosis may occur via a mechanism of angiotropic microsatellitosis leading to extravascular migratory metastasis.

- Histopathologic identification is achieved with immunohistochemistry using CD31 and D2-40 antibodies.

- The diagnosis of EVVM in conjunctival melanoma may represent a high-risk finding possibly related to increased melanoma-related mortality.

\section{Keywords}

Conjunctival melanoma · Local metastasis - Extravascular migratory metastasis · Microsatellitosis · Angiotropism

\section{Abstract \\ Purpose: To report a case of local metastasis of conjunctival melanoma, which may occur via extravascular migratory metastasis (EVMM), and discuss its clinical relevance in con-}

junctival melanoma tumor staging and possible management implications. Methods: Retrospective chart review of a single clinical case with clinicopathologic correlation. $\mathbf{R e}$ sults: A 65-year-old male referred due to local recurrence of conjunctival melanoma at the caruncle was successfully treated after two excisional procedures with negative sentinel lymph node biopsies. Forty-eight months after initial presentation, the patient developed a nodular lesion representing local recurrence in the ipsilateral upper tarsal con- karger@karger.com

(C) 2020 S. Karger AG, Basel

www.karger.com/oop

Karger ${ }^{\prime}=$
Arun D. Singh, MD

Professor of Ophthalmology, Director of Ophthalmic Oncology Cole Eye Institute and Taussig Cancer Center, Cleveland Clinic 9500 Euclid Avenue, i32, Cleveland, OH 44195 (USA)

E-Mail singha@ccf.org 
junctiva, distant from the primary tumor site. Histopathology showed nodules in the substantia propria in the absence of primary acquired melanosis. The tumor cells were found along the extravascular surface without intralymphatic or intravascular tumor cells consistent with local metastasis. One possible mechanism is angiotropic microsatellitosis leading to local EVMM. Additional neck CT imaging showed no lymphadenopathy. Conclusion: EVMM via angiotropic microsatellitosis is another possible mechanism of noncontiguous local recurrence of conjunctival melanoma. Angiotropic microsatellitosis may represent a high-risk finding possibly related to increased melanoma-related mortality.

(c) 2020 S. Karger AG, Basel

\section{Introduction}

The goals of treatment of conjunctival melanoma are to achieve local control, prevent recurrence, and minimize the risk for distant metastasis $[1,2]$. Local recurrence in conjunctival melanoma arises most commonly from remnant tumor present in margins after excision (contiguous recurrence), especially in cases of melanoma arising from primary acquired melanosis (PAM) with atypia or cases with clinically unrecognized amelanotic melanoma beyond the tumor margins [1,3-6]. A distinct entity of local conjunctival metastasis (LCM) represents a pattern of noncontiguous recurrence at a location distant from the primary site and in the absence of PAM. Previous reports have proposed intralymphatic spread as the mechanism for LCM $[7,8]$. However, histopathologic descriptions have not demonstrated evidence of intravascular or intralymphatic tumor presence in any of the cases $[7,8]$.

Melanoma cells migrating along the abluminal surface of vascular channels without intravasation (angiotropism or angiotropic microsatellitosis), leading to local metastasis, is termed extravascular migratory metastasis (EVMM). Lugassy and Barnhill first described such a mechanism of local metastasis in cutaneous melanoma $[9,10]$. Barnhill et al. [11] have recently proposed that conjunctival melanoma can also locally metastasize via EVMM.

Herein, we provide a clinicopathologic correlation, suggesting the presence of local metastasis in a case of conjunctival melanoma. In addition, we discuss clinical relevance of EVMM as a possible mechanism of local metastasis in conjunctival melanoma and its relevance for tumor staging and possible management implications.

\section{Case Report}

A 65-year-old male was referred following incomplete excision of a conjunctival melanoma arising from the right eye caruncle (Fig. 1a). Review of previous pathology showed invasive conjunctival melanoma with positive peripheral and deep margins and a maximum thickness of $4 \mathrm{~mm}$. We performed local tumor excision with $4 \mathrm{~mm}$ safety margins, partial thickness scleral dissection, adjuvant cryotherapy to the margins, and additional conjunctival map biopsies. Histopathologic diagnosis confirmed the presence of invasive conjunctival melanoma with a maximum thickness of $1.4 \mathrm{~mm}$. The deep margin was clear and one peripheral margin showed presence of melanoma in situ. There was no lymphovascular invasion, assessed by CD-31 staining, and no perineural invasion. The map biopsies were negative for PAM. Then, the patient underwent 4 cycles of topical mitomycin C (Fig. 1b).

A total of 36 months after presentation, the patient developed local recurrence with a nodular shaped, darkly pigmented conjunctival mass in the superonasal area in proximity to the previous biopsy site (Fig. 1c). We performed excisional biopsy, adjuvant cryotherapy, and episcleral dissection. Histopathologic diagnosis confirmed the presence of an epithelioid-type conjunctival melanoma with a maximum thickness of $3.7 \mathrm{~mm}$ and clear deep and lateral margins. Additional findings included the presence of ulceration, 11 mitotic figures per 10 high power fields (HPF), and no evidence of lymphovascular invasion. Mutation for BRAF was positive. There were no NRAS or KIT mutations. The patient underwent sentinel lymph node biopsy, which was negative for postauricular, inferior parotid, and level IIA and IIB lymph nodes. The patient had an unremarkable postoperative course.

Forty-eight months after his initial presentation, he complained of foreign body sensation. The caruncular and superonasal fornix areas showed no tumor recurrence (Fig. 1d). Upon right upper lid eversion, two new pigmented tarsal conjunctival lesions were located $3 \mathrm{~mm}$ from the lid margin (Fig. 1e). The lesions composed a total area measuring $5 \times 4 \mathrm{~mm}$, were noncontiguous from each other, and were located more than $15 \mathrm{~mm}$ from the previously excised caruncular tumor. We then proceeded with a partial thickness $9 \mathrm{~mm}$-wide local excision with adjuvant cryotherapy to the margins. Histopathologic diagnosis confirmed two noncontiguous foci of invasive conjunctival melanoma with a maximal thickness of $1.5 \mathrm{~mm}$. Complete excision was confirmed by clear lateral and deep margins. One of the melanoma foci appeared to represent a microsatellite nodule, located along the stromal extravascular region, but not involving the intraluminal vascular space, as confirmed by CD-31 staining (Fig. 2). Neck lymphadenopathy was not detected on a contrast-enhanced neck CT scan.

\section{Discussion}

Conjunctival melanoma is believed to achieve local metastasis via lymphatic dissemination and distant metastatic dissemination through hematogenous spread.

Our case provides histopathologic evidence of extravascular spread (EVMM) of a primary conjunctival melanoma in the absence of PAM. The noncontiguous recur- 

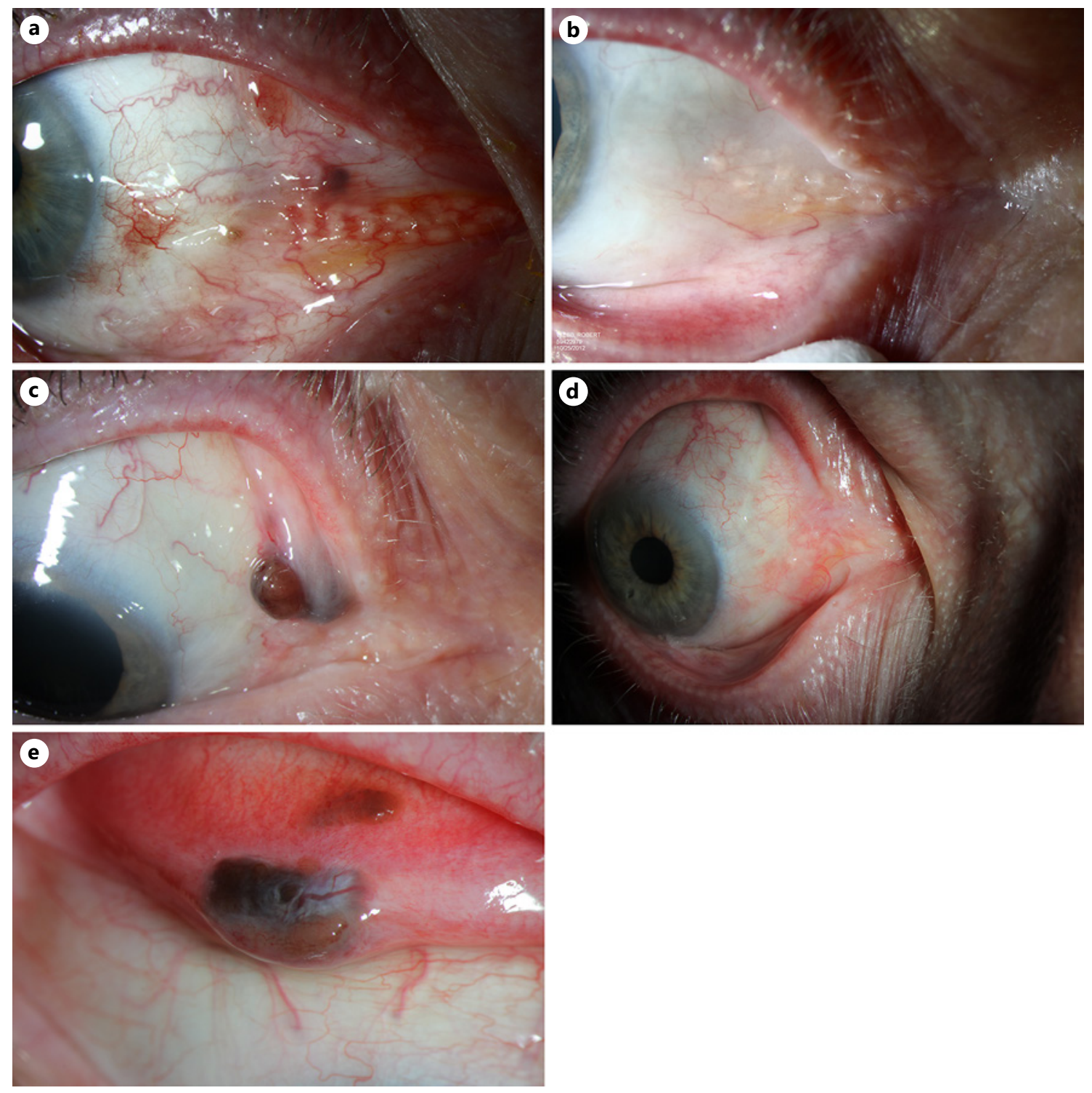

Fig. 1. The patient presented initially with an incompletely excised invasive conjunctival melanoma arising from the right caruncle (a). Clinical appearance at 9 months after wide margin excision, partial thickness scleral dissection, adjuvant cryotherapy, and topical mitomycin with clear margins in histopathology (b). Local recurrence at 36 months with a nodular shaped pigmented mass at the caruncle area (c). At 48 months, the caruncular area remained clear of tumor recurrence (d); however, the right upper tarsal conjunctiva showed multifocal nodular pigmented lesions which were excised and treated with adjuvant cryotherapy (e).

rent lesions in our case were located at the tarsal conjunctiva, distant from the primary melanoma site in the caruncle. Histopathologically, the metastatic nodules were separated from the overlying epithelium by a grenz zone of collagen, further supporting their metastatic status. We further located the metastatic melanoma cells along the abluminal surface of vascular channels in the conjunctival stroma. In addition, we showed the absence of any melanoma cells within the intraluminal space using immunohistochemistry. The local metastatic conjunctival melanoma cells located in the abluminal space in the absence of any intravascular melanoma cells may suggest angiotropic microsatellitosis as the mechanism of local EVMM. It is important to note that the use of immunohistochemistry with CD31 and D2-40 facilitates the localization of the migratory melanoma cells in rela- 

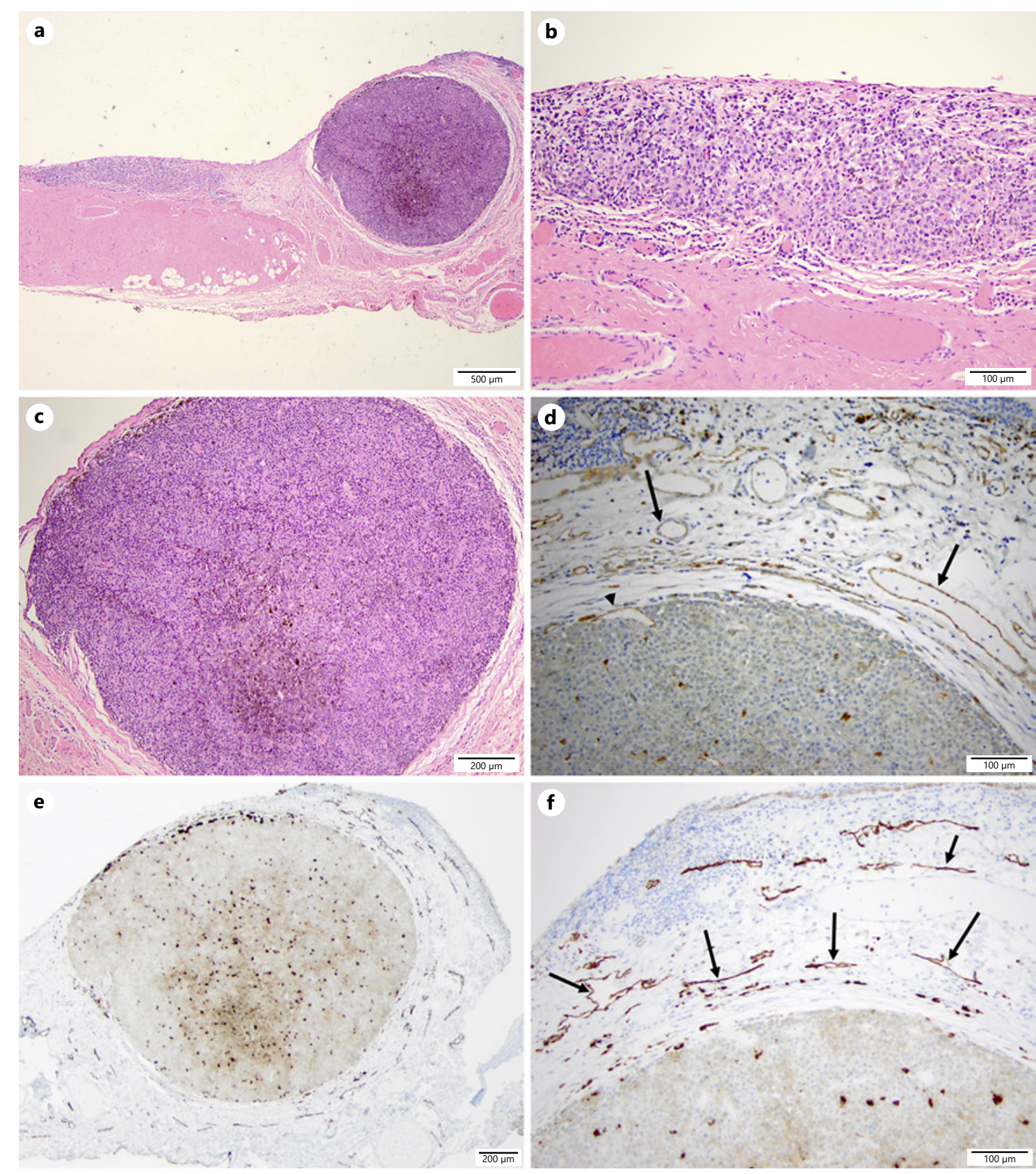

Fig. 2. Low magnification (a) and high magnification (b, c) H\&E histologic images of the excised right upper tarsal conjunctival lesions showing two distinct micronodules with clear lateral and deep margins. CD31 highlights small vessels within the tumor (arrowhead) and in the surrounding substantia propria (arrows) but no circumferential vessel wall around the nodule (d). D2-40 confirms that there is no lymphovascular wall around the satellite nodule. Several small vessels in the substantia propria appear compressed by the expanding neoplasm (e). Higher magnification view of lymphovascular spaces marked by D2-40 (f).

tion to intravascular and intralymphatic channels. Standard histopathologic analysis with $\mathrm{H} \& \mathrm{E}$ alone is not sufficient to identify the mechanism of angiotropic microsatellitosis in conjunctival melanoma.
Jakobiec et al. [8] reported 2 patients in which local metastasis occurred in locations distant from their primary sites. The authors proposed that local conjunctival "in-transit" metastasis may have occurred via intralym- 
phatic spread with additional distant metastasis and subsequent melanoma-related death in one case. Histologically, both cases showed a grenz zone of collagen separating the epithelium from the underlying nodule of locally metastatic melanoma, suggesting that the metastatic lesions did not arise from local PAM. However, their report lacked histological evidence of intralymphatic or intravascular tumor deposits in both cases and did not provide a mechanistic explanation for these events.

Mudhar and Rennie [7] have explored alternative mechanisms for local metastasis of conjunctival melanoma. In a series of 7 patients, they reported two distinct histopathologic types; diffuse along the bulbar conjunctiva and nodular in the fornix and tarsal conjunctiva. All samples were found to be extravascular and did not show histopathologic evidence of intraluminal deposits of melanoma cells with CD31 (vascular endothelial marker) and D2-40 (lymphatic endothelial marker) immunohistochemical stains. In addition, lymphatic channels were present within and adjacent to the metastasis. These observations led the authors to postulate conjunctival melanoma-triggered lymphangiogenesis [12-14]. However, the lack of histopathologic evidence of intraluminal melanoma cells may be representative of EVMM.

Identifying angiotropic microsatellitosis as a mechanism of local metastasis in conjunctival melanoma has several clinical implications. From a chronobiological standpoint, LCM would precede regional lymph node spread and eventual distant organ involvement. The AJCC staging system has recognized microsatellite nodules as a high-risk pathologic finding, possibly related to increased melanoma-related mortality. In their case series, Barnhill et al. [11] reported that 4 out of 5 patients with conjunctival melanoma microsatellitosis had a higher risk for lymph node metastasis, distant metastasis, and death. Retrospective studies have validated the most recent AJCC staging system and have showed that recurrence and metastasis rates increase progressively as the stage of conjunctival melanoma progresses $[15,16]$. Although the histopathologic presence of EVMM is recognized as a high-risk feature, it has yet to be included in the criteria representing " $\mathrm{N}$ " status for conjunctival melano- ma in the AJCC TNM staging classification system [7]. Adopting local metastasis as " $N$ " status, regardless of the mechanism (intralymphatic or EVMM), will influence the surveillance strategy $[1,17]$ and may also identify patients that would most likely benefit from adjuvant therapy $[18,19]$.

\section{Conclusion}

Local noncontiguous recurrence of conjunctival melanoma in the absence of PAM may occur as EVMM via angiotropic microsatellitosis. Histopathologic identification is achieved with immunohistochemistry using CD31 and D2-40 antibodies. The diagnosis of EVMM in conjunctival melanoma may represent a high-risk finding possibly related to increased melanoma-related mortality.

\section{Statement of Ethics}

Our case report complies with the guidelines for human studies and was conducted ethically in accordance with the World Medical Association Declaration of Helsinki.

\section{Disclosure Statement}

The authors have no conflicts of interest to declare.

\section{Funding Sources}

The authors have no additional funding sources to disclose.

\section{Author Contributions}

J.J.E., G.Y., R.C., and A.D.S. collected data, wrote the manuscript, prepared figures, and critically edited the article. All named authors meet the International Committee of Medical Journal Editors (ICMJE) criteria for authorship for this article, take responsibility for the integrity of the work as a whole, and have given their approval for this version to be published.

\section{References}

Conjunctival Melanoma Microsatellitosis
1 Aziz HA, Gastman BR, Singh AD. Management of Conjunctival Melanoma: Critical Assessment of Sentinel Lymph Node Biopsy. Ocul Oncol Pathol. 2015 Jun;1(4):266-73

2 Esmaeli B, Wang X, Youssef A, Gershenwald JE. Patterns of regional and distant metastasis in patients with conjunctival melanoma: experience at a cancer center over four de- cades. Ophthalmology. 2001 Nov; 108(11): 2101-5

3 Shields CL, Shields JA, Gündüz K, Cater J, Mercado GV, Gross N, et al. Conjunctival melanoma: risk factors for recurrence, exenteration, metastasis, and death in 150 consecutive patients. Arch Ophthalmol. 2000 Nov; 118(11):1497-507. 
4 Missotten GS, Keijser S, De Keizer RJ, De Wolff-Rouendaal D. Conjunctival melanoma in the Netherlands: a nationwide study. Invest Ophthalmol Vis Sci. 2005 Jan;46(1):75-82.

5 Tuomaala S, Eskelin S, Tarkkanen A, Kivelä T. Population-based assessment of clinical characteristics predicting outcome of conjunctival melanoma in whites. Invest Ophthalmol Vis Sci. 2002 Nov;43(11):3399-408.

6 Triay E, Bergman L, Nilsson B, All-Ericsson $\mathrm{C}$, Seregard S. Time trends in the incidence of conjunctival melanoma in Sweden. Br J Ophthalmol. 2009 Nov;93(11):1524-8.

7 Mudhar HS, Rennie IG. Local conjunctival metastases from primary conjunctival melanoma: clinico-pathological correlation and implications. Br J Ophthalmol. 2013 Jan; 97(1):33-9.

8 Jakobiec FA, Buckman G, Zimmerman LE, La Piana FG, Levine MR, Ferry AP, et al. Metastatic melanoma within and to the conjunctiva. Ophthalmology. 1989 Jul;96(7):9991005.

9 Lugassy C, Barnhill RL, Christensen L. Melanoma and extravascular migratory metastasis. J Cutan Pathol. 2000 Oct;27(9):481.
10 Barnhill RL, Lugassy C. Angiotropic malignant melanoma and extravascular migratory metastasis: description of 36 cases with emphasis on a new mechanism of tumour spread. Pathology. 2004 Oct;36(5):485-90.

11 Barnhill RL, Lemaitre S, Lévy-Gabrielle C, Rodrigues M, Desjardins L, Dendale R, et al. Satellite in transit metastases in rapidly fatal conjunctival melanoma: implications for angiotropism and extravascular migratory metastasis (description of a murine model for conjunctival melanoma). Pathology. 2016 Feb;48(2):166-76.

12 Zimmermann P, Dietrich T, Bock F, Horn FK, Hofmann-Rummelt C, Kruse FE, et al. Tumour-associated lymphangiogenesis in conjunctival malignant melanoma. Br J Ophthalmol. 2009 Nov;93(11):1529-34.

13 Heindl LM, Hofmann-Rummelt C, Adler W, Bosch JJ, Holbach LM, Naumann GO, et al. Tumor-associated lymphangiogenesis in the development of conjunctival melanoma. In vest Ophthalmol Vis Sci. 2011 Sep;52(10): 7074-83.

14 Heindl LM, Hofmann-Rummelt C, Adler W, Bosch JJ, Holbach LM, Naumann GO, et al. Prognostic significance of tumor-associated lymphangiogenesis in malignant melanomas of the conjunctiva. Ophthalmology. 2011 Dec;118(12):2351-60.
15 Yousef YA, Finger PT. Predictive value of the seventh edition American Joint Committee on Cancer staging system for conjunctival melanoma. Arch Ophthalmol. 2012 May; 130(5):599-606.

16 Shields CL, Kaliki S, Al-Dahmash SA, Lally SE, Shields JA. American Joint Committee on Cancer (AJCC) clinical classification predicts conjunctival melanoma outcomes. Ophthal Plast Reconstr Surg. 2012 Sep-Oct;28(5):31323.

17 Savar A, Esmaeli B, Ho H, Liu S, Prieto VG. Conjunctival melanoma: local-regional control rates, and impact of high-risk histopathologic features. J Cutan Pathol. 2011 Jan;38(1): 18-24.

18 Long GV, Hauschild A, Santinami M, Atkinson V, Mandalà M, Chiarion-Sileni V, et al. Adjuvant Dabrafenib plus Trametinib in Stage III BRAF-Mutated Melanoma. N Engl J Med. 2017 Nov;377(19):1813-23.

19 Weber J, Mandala M, Del Vecchio M, Gogas HJ, Arance AM, Cowey CL, et al.; CheckMate 238 Collaborators. Adjuvant Nivolumab versus Ipilimumab in Resected Stage III or IV Melanoma. N Engl J Med. 2017 Nov;377(19): 1824-35. 